\title{
Biofilm Inactivation and Prevention on Common Implant Material Surfaces by Nonthermal DBD Plasma Treatment
}

\author{
Fatma İbiş, Hakan Oflaz, \& Utku Kürşat Ercan* \\ Department of Biomedical Engineering, İzmir Katip Çelebi University, İzmir, Turkey \\ *Address all correspondence to: Utku Kürşat Ercan, İzmir Katip Çelebi University, Çiğli Ana Kampüsü, 35620 Çiğli/ \\ İzmir, Turkey; Tel.: +90 232329 3535/3747 or +90 535946 0660; Fax: +90 232386 0888, E-mail: \\ utkuercan@gmail.com, utkuk.ercan@ikc.edu.tr
}

\begin{abstract}
Two stainless steels (SSs) - 304 and 316L—as well as a titanium-aluminum-vanadium alloy (Ti6A14V) and ultra-high-molecular-weight polyethylene (UHMWPE) are common metallic and polymeric materials used in medicine for various applications such as bone fracture fixation, dental and cardiac implants, production of medical devices. Implanted materials are highly susceptible to infections that lead may implant failure. The method used to sterilize implant materials plays an important role in the success and performance of an implant. Mechanical properties of UHMWPE are compromised as a consequence of gamma irradiation. Repetitive autoclaving of implants and surgical tools reduces material performance. Also, custom orthopedic prosthesis production requires cheap, on-site, and quick sterilization procedures. In this study we evaluated the inactivation and prevention of biofilm formation of Escherichia coli and Staphylococcus aureus on UHMWPE, a Ti6Al4V, 304 SS, and 316L SS surfaces. Plasma treatment of discs was evaluated by an XTT (2,3-bis-(methoxy-4-nitro-5-sulfophenyl)- 2H-tetrazolium-5-carboxanilide]) viability assay, which showed that $95 \%$ of both bacterial biofilms were inactivated after 3 minutes of plasma treatment. For the biofilm prevention tests, UHMWPE, Ti6Al4V, 304 SS, and 316L SS discs were first treated with nonthermal dielectric barrier discharge plasma; then, 1-day-old biofilms of E. coli and S. aureus were grown on the disc surfaces, and biofilm formation was similarly evaluated using an XTT assay. Plasma treatment of implant surfaces prevents biofilm formation up to $50 \%$. Safranin assay, which was used to evaluate extracellular polymeric substances, also has shown that plasma treatment of UHMWPE, Ti6A14V, 304 SS, and 316L SS discs not only inactivates biofilms but also but also disrupts extracellular polymeric substances that are secreted by bacteria during biofilm growth.
\end{abstract}

KEY WORDS: biofilm, biofilm prevention, implant, nonthermal plasma

\section{INTRODUCTION}

Metals, as medical devices and implants, have been on the scene of medicine for long time, since the first report by Lane in 1895 for bone fracture fixation. Various metallic materials are now being used to fabricate various medical devices and implants that are used in different parts of the body. ${ }^{1}$ For example, $316 \mathrm{~L}$ stainless steel (SS) and a titanium-aluminum-vanadium alloy (Ti6A14V) are used in the fabrication of cardiac implants such as stents and artificial heart valves, orthopedic implants and total joint prosthesis, dental implants and orthodontic wires, and artificial eardrums. In addition, $304 \mathrm{SS}$ is used in the production of hand tools and trial prostheses. Ultra-high-molecular-weight polyethylene (UHMWPE) is used for acetabular cups and inserts in total knee prostheses. ${ }^{2}$ 
The sterility of implants is a common concern. ${ }^{3}$ Well-established sterilization methods such as autoclaving and gamma irradiation are routinely performed to sterilize metallic materials. Moreover, ethylene oxide and gamma irradiation sterilization techniques are used to sterilize UHMWPE. However, ethylene oxide sterilization leaves toxic remnants, and gamma irradiation causes changes in the chemical and mechanical properties of UHMWPE, which adversely affect the life span of UHMWPE implants. ${ }^{4}$ Moreover, with developing metal manufacturing methods such as sintering, it is currently possible to produce patient-specific prosthesis and implants using 3-dimensional printing; this increases the importance of fast, onsite sterilization techniques that could be applied shortly before implantation. ${ }^{5}$

Compromised skin during implantation surgery is considered to be a cause of implant-related surgical site infection. Moreover, since the implanted prostheses and implants are foreign materials, they serve as excellent surfaces for bacterial adherence and biofilm formation, which lead to surgical site infections that reduce the performance of implants and prostheses and increase both the healing period and health care expenses. ${ }^{6,7}$

Biofilm is a group of microorganisms in which cells adhere to each other on living and/or nonliving surfaces. ${ }^{7,8}$ Biofilm formation is a complex process that involves a series of steps. Reversible adherence of planktonic cells to a surface triggers biofilm formation. ${ }^{9}$ During biofilm formation, cells secrete extracellular polymeric substances (EPSs) whose main constituents are polysaccharides and proteins, along with relatively smaller concentrations of nucleic acids, lipids, and other biological macromolecules. Presence of EPSs plays a major role in the interactions between bacterial cells and with the surface to adhere and shift bacteria into a biofilm-specific physiological state. ${ }^{9}{ }^{10}$ EPSs provide structural support and increases nutrient uptake; they also act as a barrier and protect organisms in the biofilm against common antimicrobial substances. Therefore biofilms are up to 1000 times more resistant to antimicrobial substances, including antibiotics. Biofilm formation on medical devices and on implant materials is a common concern for the control and prevention of implant-related and surgical site infections. ${ }^{7,11,12}$ Periimplantitis is a common example of implant-related infection in dentistry that may lead to bone loss at the mandible; it requires the eradication of the biofilm from the implant surface. Mechanical techniques are mostly used for the treatment of peri-implantitis but show limited efficiency. Thus additional biocidal methods, such as the use of antibiotics and antiseptics, are proposed to be combined with mechanical techniques. Antibiotics and other antiseptic agents may remain ineffective in the treatment of peri-implantitis since it is mainly caused by resistant bacteria, similar to other implant-related and surgical site infections. ${ }^{11-13}$

Antimicrobial activity of nonthermal plasma is well known, and its effects on various microorganisms, including bacteria, fungi, viruses, and even prions, have been demonstrated. ${ }^{14-17}$ Not only does nonthermal plasma have antimicrobial activity, but materials treated with nonthermal plasma, such as different liquids and gels, acquire a strong antimicrobial activity that is effective on both the planktonic and biofilm forms of microorganisms. ${ }^{18-20}$ Moreover, Monetta et al. ${ }^{21}$ showed that a titanium alloy treated 
with nonthermal oxygen plasma acquired antimicrobial activity, and the effect persisted up to 16 days.

Biofilm formation on metallic and polymeric implant materials and medical devices is a significant public health problem and causes risk for both patients and health care providers. An effective method for inactivation and prevention of biofilm is crucial. This study evaluates the use of atmospheric pressure, nonthermal dielectric barrier discharge (DBD) air plasma treatment for biofilm eradication and prevention on $304 \mathrm{SS}, 316 \mathrm{~L} \mathrm{SS}$, Ti6A14V, and UHMWPE surfaces.

\section{MATERIALS AND METHODS}

Medical-grade 304 SS, 316 SS, Ti6Al4V (grade 5 titanium), and UHMWPE rods were generously donated by Hipokrat Medical Devices A.Ş. (İzmir, Turkey). The rods were cut to discs (10 $\mathrm{mm}$ diameter, $3 \mathrm{~mm}$ thickness).

Nonthermal plasma was generated using an alternating current (AC) microsecondpulsed power supply (Advanced Plasma Solutions, Malvern, PA). A custom-made, rectangular DBD electrode was fabricated in our laboratory using a 10-mm-thick copper plate (64 mm long, $38 \mathrm{~mm}$ wide). The surface of the cooper plate was covered with a tempered, 1 -mm-thick glass slide $(50 \times 75 \mathrm{~mm})$ that served as a dielectric barrier. The remainder of the copper plate was insulated with polyethylene housing. Discs were treated with air DBD plasma at atmospheric pressure. The discharge gap between the DBD high0voltage electrode and the surfaces of the discs was maintained at $2 \mathrm{~mm}$ (Fig. 1). The microsecond plasma power supply was operated at a $1.5-\mathrm{kHz}$ frequency, a $31-\mathrm{kV}$ peak-to-peak voltage, and a $10-\mu$ s pulse duration, which yields a power density of $0.29 \mathrm{~W} / \mathrm{cm}^{2}$.

First, the zone of inhibition (ZOI) was tested to evaluate whether plasma-treated implant surfaces acquire antimicrobial activity. In this test, $1 \mathrm{~mL}$ of $10^{7}$ colony-forming

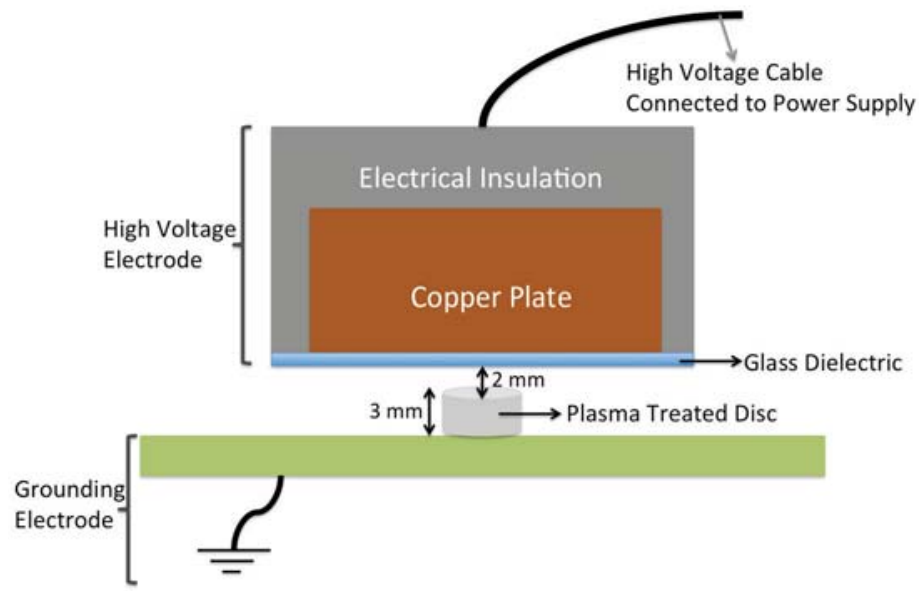

FIG. 1: Schematic of the dielectric barrier discharge plasma electrode and plasma treatment setup. Note that the plasma discharge gap was set to $2 \mathrm{~mm}$ over the surface of 3-mm-thick discs.

Volume 6, Issue 1, 2016 
İbiş, Oflaz, \& Ercan

units (CFUs)/mL Escherichia coli suspension was spread on trypticase soy agar (TSA) plates, and left to dry under aseptic conditions. After the bacteria suspension was dried, discs whose surfaces had been treated with DBD plasma for 10 minutes were placed on TSA plates and incubated overnight. Untreated discs were used as a positive control. After 24 hours of incubation, a bacterial lawn was grown on the TSA plates; this presents as a sticky surface and can be removed mechanically when it comes into contact with another surface. Thus, following overnight incubation, discs were removed and plates were incubated for an additional 24 hours to ensure that an inhibition zone was formed by only an antimicrobial effect on the surface of the disc, rather than the mechanical removal of bacteria during removal of the discs. After this additional 24 hours of incubation, the inhibition zone was evaluated.

The antimicrobial activity of plasma-treated disc surfaces also was evaluated with a colony-counting assay. All experiments were performed in triplicate. Similar to the ZOI test, discs of implant materials were treated for 10 minutes at $1.5 \mathrm{kHz}$ and $31 \mathrm{kV}$. E. coli and Staphylococcus aureus were used as model gram-negative and gram-positive organisms, respectively. Following plasma treatment, $100 \mu \mathrm{L}$ of $10^{5} \mathrm{CFUs} / \mathrm{mL}$ bacterial suspension, which yields a final bacterial number of $10^{4} \mathrm{CFUs}$, were added to the discs and held for 30 minutes. After 30 minutes of holding time, bacteria were serially diluted using sterile $1 \mathrm{X}$ phosphate-buffered saline (PBS) and spread on the TSA plates. Plates were incubated at $37^{\circ} \mathrm{C}$ overnight, and surviving colonies were counted after incubation.

Contact angles were measured using a KSV Attension Theta goniometer (Biolin Scientific, Stockholm, Sweden). All measurements were done in triplicate. A distilled water droplet $(4 \mu \mathrm{L})$ was dispensed on the surfaces of plasma-treated and untreated (control) discs. The water droplet was analyzed by the system, in which 120 measurements were taken from the left and right sides of the droplet. The contact angle was given as the mean of all measurements from the left and right sides.

Eradication of the biofilm and prevention of biofilm formation was assessed using XTT XTT (2,3-bis-(methoxy-4-nitro-5-sulfophenyl)- 2H-tetrazolium-5-carboxanilide] and safranin assays. All experiments were performed in triplicate. For biofilm eradication experiments, the bacterial suspension was incubated overnight in a shaker incubator $\left(120 \mathrm{rpm}, 37^{\circ} \mathrm{C}\right)$. Culture $(100 \mu \mathrm{L})$ grown overnight and $100 \mu \mathrm{L}$ of $50 \% \mathrm{v} / \mathrm{w}$ glucose solution, as a biofilm enhancer, were added to $10 \mathrm{~mL}$ of tryptic soy broth (TSB) to prepare the biofilm growth medium. Each disc was made of a different implant material (Ti6Al4V, 304 SS, 316L SS, or UHMWPE); the discs were placed into a 12-well plate, and $1 \mathrm{~mL}$ of biofilm growth medium was added to each disc, then incubated for 24 hours in a stationary incubator at $37^{\circ} \mathrm{C}$. After 24 hours of biofilm growth, discs were washed twice using $1 \mathrm{X}$ PBS to remove nonadherent bacteria, and each disc was treated for 1, 2, and 3 minutes with nonthermal, atmospheric DBD plasma $(1.5 \mathrm{kHz}, 31 \mathrm{kV})$. After plasma treatment, discs were washed for once using sterile $1 \mathrm{X}$ PBS, then $600 \mu \mathrm{L}$ of 0.5 $\mathrm{mg} / \mathrm{mL}$ XTT was added to each disc and incubated for 4 hours at $37^{\circ} \mathrm{C}$. After incubation of XTT, $100 \mu \mathrm{L}$ of reacted XTT, which develops an orange color as the result of cellular respiration, was transferred to a 96-well plate; absorbance was measured at 496 
$\mathrm{nm}$. Untreated discs were used as a positive control. Measurement of the absorbance on control discs was set as $100 \%$ survival, and the percentage survival of treated samples was normalized to the control group.

During biofilm formation, cells secrete an EPS that provides an adherence medium to enhance the structural integrity of the bacteria in the developing biofilm. In biofilm eradication tests, safranin assay, which binds to EPSs, was used to visualize and quantify the EPS. One-day-old biofilms were grown on the discs, as mentioned earlier. After the growth of the biofilm, discs were washed twice using sterile 1X PBS to remove nonadherent bacteria, then each disc was treated for 1, 2, and 3 minutes with nonthermal, atmospheric DBD plasma $(1.5 \mathrm{kHz}, 31 \mathrm{kV})$. After plasma treatment, discs were washed once using sterile $1 \mathrm{X}$ PBS; then $600 \mu \mathrm{L}$ of $0.1 \%$ safranin was added to stain the biofilm on the discs, and the discs were held for 30 minutes at room temperature. Afterward, the safranin dye was removed by pipetting and the biofilms were left to dry. Once the biofilms were dry, $450 \mu \mathrm{L}$ of $30 \%$ acetic acid was added to dissolve the safranin dye, and $100 \mu \mathrm{L}$ of dissolved safranin was transferred to a 96-well plate. Absorbance was measured at $550 \mathrm{~nm}$.

For biofilm prevention experiments, discs made of different implant materials were first treated for 10 minutes with nonthermal, atmospheric DBD plasma $(1.5 \mathrm{kHz}, 31$ $\mathrm{kV})$. All experiments are performed in triplicate. After plasma treatment, $1 \mathrm{~mL}$ of biofilm growth medium was promptly added to the plasma-treated discs and they were incubated at $37^{\circ} \mathrm{C}$ for 24 hours to evaluate 1-day-old biofilm development. After 24 hours of incubation, discs were washed twice using sterile 1 X PBS, and XTT and safranin assays were carried out in a similar manner as performed in the biofilm eradication experiments.

\section{RESULTS AND DISCUSSION}

In ZOI experiments, after 24 hours of incubation both plasma-treated and untreated (control) discs were removed to inspect the ZOI. These experiments revealed the presence of ZOIs where both plasma-treated and untreated control discs were placed after 24 hours of incubation. It was thought that such an observation could be a result of the mechanical removal of surviving bacteria in the control group. Therefore, to monitor the inhibition zone, plates were incubated for an additional 24 hours to let surviving residual bacteria grow after removal of both the plasma-treated and untreated (control) discs and to ensure that the inhibition zones are not only present as a result of the mechanical removal of the discs. Following the second 24 hours of incubation, bacterial growth was observed where untreated discs were placed, and there was no bacterial growth on the areas where plasma-treated discs were placed (Fig. 2). At this point, a question regarding the presence of inhibition zones may arise because of the lack of oxygen where the disc comes into contact with bacteria. However, E. coli is a facultative anaerobic bacteria and can grow in the absence of oxygen. Therefore the presence of inhibition zones caused by oxygen deprivation could be ruled out and might be attributed to antimicrobial activity that was acquired by discs following plasma treatment.

Volume 6, Issue 1, 2016 


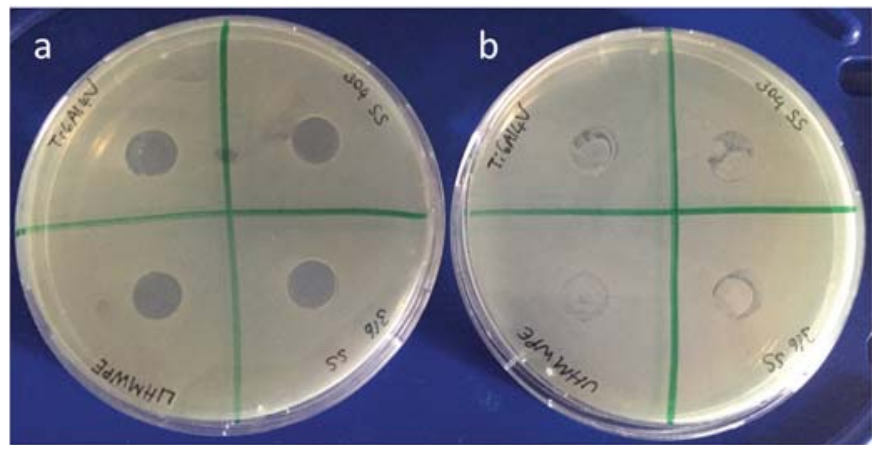

FIG. 2: Inhibition zone on where plasma-treated (A) and untreated control (B) discs were placed. Both the control and plasma-treated discs were incubated for 24 hours. To ensure that the inhibition zone was not only created by the mechanical removal of the organism, after removal of the discs the plates were incubated 24 hours more to let the remaining organism grow. After a total of 48 hours of incubation, an inhibition zone where plasma-treated discs were placed (A) and bacterial growth where untreated control discs were placed (B) was observed.

The colony-counting assay showed that surfaces of metallic implant materials treated with plasma for 10 minutes acquire antimicrobial activity. As depicted in Fig. 3, 304 SS showed 4-log inactivation of both E. coli and S. aureus, whereas 316L SS and Ti6A14V showed about 4-log and 3-log inactivation on E. coli and S. aureus, respectively. On the other hand, the UHMWPE surface treated with plasma for 10 minutes showed 1-log inactivation of $S$. aureus and less than 1-log inactivation of $E$. coli. Acquisition of less antimicrobial activity by UHMWPE compared with metallic materials was attributed to its dielectric properties. The DBD plasma discharge setup consists of two conductive electrodes; at least one of these electrodes should be covered with a dielectric material. ${ }^{22}$ The DBD plasma discharge formation depends on several factors, including the type and thickness of the dielectric material. ${ }^{23}$ The total transferred charge for plasma formation is proportional to the ratio of the relative permittivity to the thickness of the dielectric material. ${ }^{24}$ When a thicker dielectric material is used to cover the surface of the electrodes, the threshold voltage that is required for ionization of gas (or plasma formation) should be increased..$^{25}$ In this study all discs were placed on a counter, grounding electrode. Because of the lower conductivity of UHMWPE, it served as a thick dielectric material covering the grounding electrode, while the surfaces of the metal discs served as the counterelectrode. Thus plasma discharge generated over the UHMWPE disc was lower, which led to less antimicrobial activity.

The antimicrobial activity of cold plasma is well known and widely reported. ${ }^{26}$ In addition to the antimicrobial activity of cold plasma, various plasma-treated liquids such as water, $\mathrm{N}$-acetylcysteine, sodium chloride, and PBS solution can acquire antimicrobial properties. ${ }^{18,27,28}$ Moreover, various materials treated by plasma can attain antimicrobial activity. Poor et al. ${ }^{19}$ showed that calcium alginate gels could acquire antimicrobial activity when treated with nonthermal plasma. This activity remains up to 21 days and is 


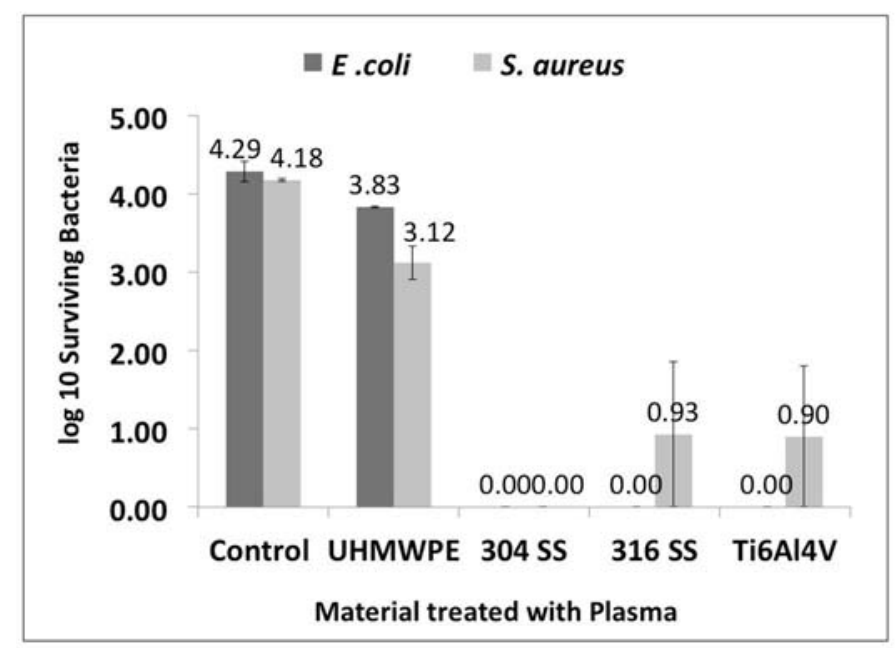

FIG. 3: Antimicrobial activity acquired by discs after a 10-minute treatment with nonthermal, atmospheric dielectric barrier discharge air plasma was tested on planktonic forms of Escherichia coli and Staphylococcus aureus. SS, stainless steel; UHMWPE, ultra-high-molecular-weight polyethylene.

effective on various pathogens. Similarly, Yorsaeng et al. ${ }^{20}$ reported that latex gloves acquire antimicrobial activity after DBD plasma treatment. In addition, Monetta et al. ${ }^{21}$ demonstrated antimicrobial activity of grade 2 titanium after oxygen plasma treatment, which persisted up to 16 days. Our results also suggest that various implant materials can attain antimicrobial activity following atmospheric air, cold DBD plasma treatment, which could be considered a novel method for the prevention of surgical site infections.

Eradication of biofilms on implant material surfaces was evaluated using XTT and safranin assays. XTT assay results showed that 1-day-old biofilms of E. coli and $S$. aureus could be effectively eradicated on 304 SS, 316L SS, Ti6Al4V, and UHMWPE surfaces. After a 3-minute plasma treatment of all materials tested in this study, about $95 \%$ of $E$. coli and $S$. aureus biofilms were inactivated. It is interesting that there was no significant difference in the inactivation rate of bacteria between the samples treated with plasma for 1, 2, and 3 minutes (Fig. 4A, B). Safranin assay that is used to evaluate the EPS secreted by biofilm-forming organisms showed that plasma treatment of $E$. coli and $S$. aureus biofilms not only inactivates cells in the biofilm but also disrupts the biofilm's structural integrity. A reduction in the absorbance of safranin that was collected from 3-minute plasma-treated surfaces was observed as an indicator of removal of the EPS. For both E. coli and $S$. aureus biofilms, EPS was significantly removed, as shown in Fig. 4C and D, respectively. Various researchers also previously reported the inactivation of biofilms on inanimate surfaces using cold atmospheric plasma. ${ }^{29-31}$ Koban et al. ${ }^{32}$ evaluated the antibacterial effect on biofilms that are formed on titanium discs as an alternative to chlorhexidine for the treatment of peri-implant mucositis. Similar

Volume 6, Issue 1, 2016 

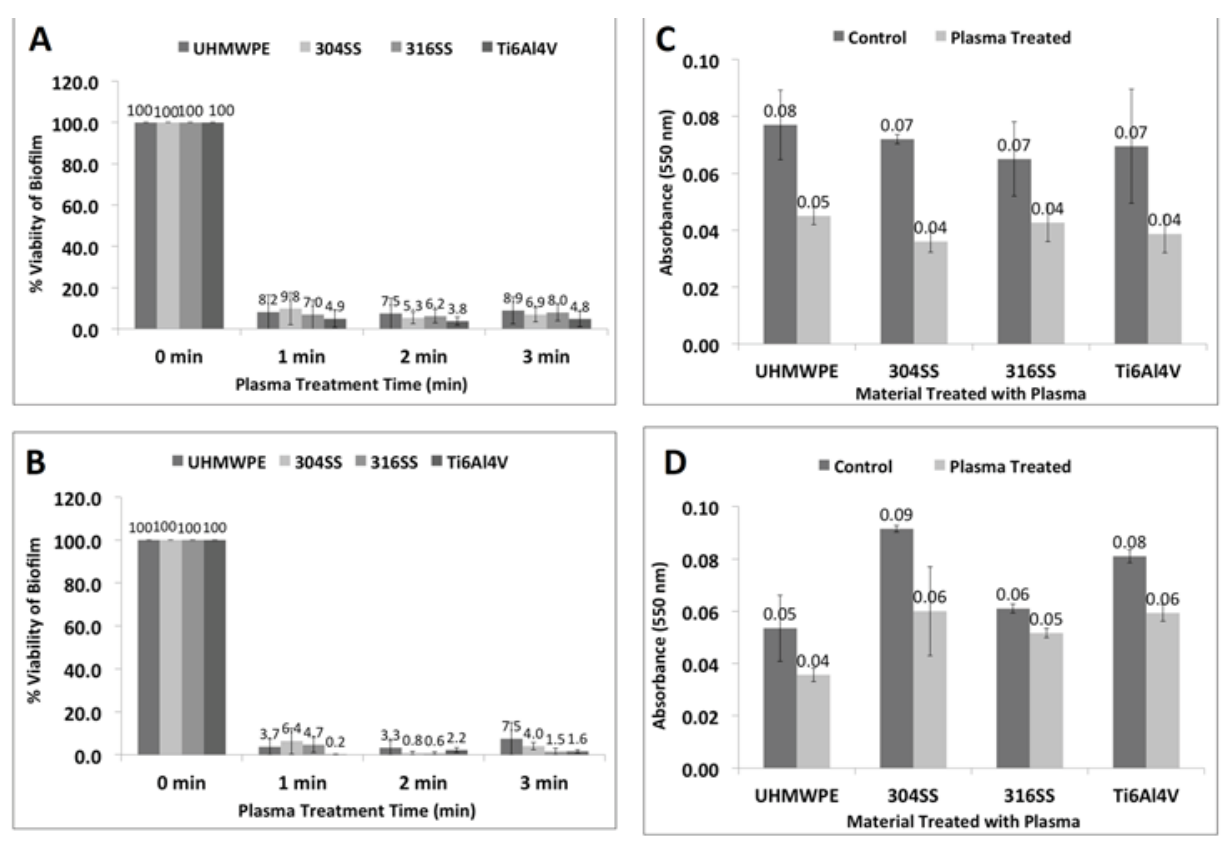

FIG. 4: Eradication of 1-day-old Escherichia coli (A) and Staphylococcus aureus (B) biofilms were evaluated using an XTT assay; the percentage of viability is represented. Nonthermal, atmospheric dielectric barrier discharge air plasma treatment inactivates up to $95 \%$ of $E$. coli and $S$. aureus biofilms, and inactivation rates are not dependent on plasma treatment duration. A safranin assay was used to monitor extracellular polymeric substances of E. coli (C) and S. aureus (D) biofilms following 3-minute discs; this not only inactivates the organism but also disrupts the integrity of the biofilm. SS, stainless steel; UHMWPE, ultra-high-molecular-weight polyethylene.

to previous reports, our results suggest that nonthermal atmospheric plasma could be introduced as a novel and effective method for decontaminating biofilms that form on medical devices. Furthermore, it also can be introduced into clinical practice for the treatment of peri-implantitis.

Contact angle measurements revealed that 10-minute plasma treatment increases the wettability of $304 \mathrm{SS}, 316 \mathrm{~L} \mathrm{SS}$, Ti6Al4V, and UHMWPE surfaces. The contact angle on implant surfaces before and after plasma treatment were measured as $100^{\circ} \pm 3^{\circ}$ and $6^{\circ} \pm 2^{\circ}$ for $304 \mathrm{SS}, 109^{\circ} \pm 4^{\circ}$ and $37^{\circ} \pm 2^{\circ}$ for $316 \mathrm{~L} \mathrm{SS}, 92^{\circ} \pm 5^{\circ}$ and $28^{\circ} \pm 3^{\circ}$ for Ti6A14V, and $86^{\circ} \pm 5^{\circ}$ and $21^{\circ} \pm 2^{\circ}$ for UHMWPE, respectively (Fig. 5).

XTT assay was also used to evaluate the prevention of E. coli and $S$. aureus biofilm formation on 10-minute plasma-treated $304 \mathrm{SS}, 316 \mathrm{~L} \mathrm{SS}$, Ti6A14V, and UHMWPE disc surfaces. Biofilm prevention experiments showed that 10-minute plasma treatment of discs before biofilm growth reduces $E$. coli biofilm formation by $64 \%, 56 \%, 27 \%$, and $22 \%$ and $S$. aureus biofilm formation by $33 \%, 47 \%, 48 \%$, and $48 \%$ on $304 \mathrm{SS}, 316 \mathrm{~L} \mathrm{SS}$, Ti6A14V, and UHMWPE disc surfaces, respectively (Fig 6A, B). Moreover, similar to 


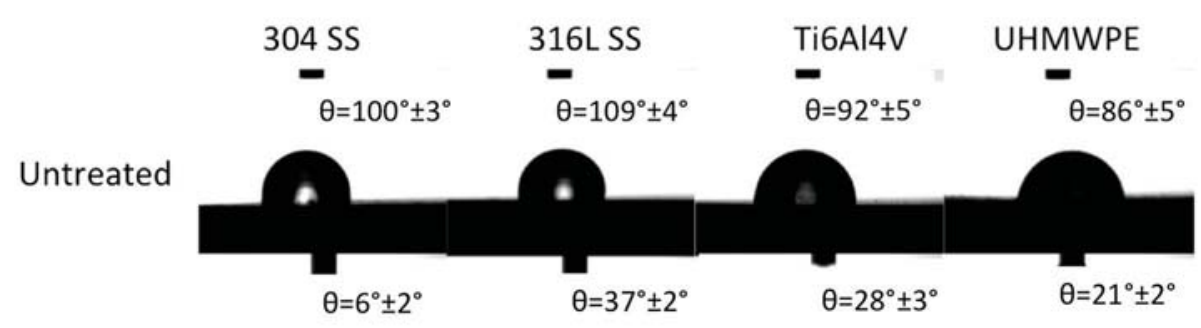

Treated

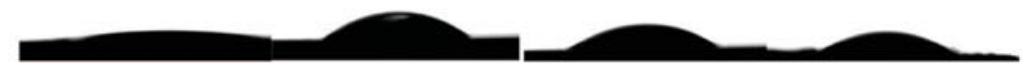

FIG. 5: Water contact angle measured on discs before and after 10-minute nonthermal, atmospheric dielectric barrier discharge air plasma treatment. Plasma treatment increases the hydrophilicity of 304 stainless steel (SS), $316 \mathrm{~L} \mathrm{SS}, \mathrm{Ti}_{6} \mathrm{Al}_{4} \mathrm{~V}$, and ultra-high-molecular-weight polyethylene (UHMWPE) surfaces.

XTT assay results, safranin assay also showed reduced biofilm formation of $E$. coli and S. aureus on plasma-treated $304 \mathrm{SS}, 316 \mathrm{~L}$ SS, Ti6Al4V, and UHMWPE disc surfaces (Fig. 6C, D). As explained previously, during biofilm formation, bacteria secrete an EPS in which cells attach to each other and adhere to the surface. Safranin dye stains EPS a reddish color and can be used to quantify secreted EPS during biofilm formation as an indicator of the biofilm's structural integrity. In other words, a darker red stain on discs represents more intense biofilm growth. In Fig. 7, discs with no biofilm formation (the negative control), with biofilm formation, and with no plasma treatment (the positive control) are represented, along with discs used in the biofilm eradication and biofilm prevention experiments. As shown in Fig. 7, the red safranin stain is substantially reduced on the discs that were used in the biofilm eradication and prevention experiments for both $E$. coli and $S$. aureus. These macroscopic observations are in correlation with the results in presented Fig. 6, which proves that plasma treatment is capable of disrupting the EPS when it is used for biofilm eradication and prevention purposes.

Safranin staining results suggest that the plasma treatment of biofilm inactivates bacteria and removes EPSs from the biofilm during the eradication of the biofilm that forms on the disc surface. EPS formation during biofilm growth is also prevented when discs are treated with cold plasma before biofilm growth.

Even though hydrophobic surfaces are considered to assist bacterial cell adherence during biofilm formation, there is no explicit consensus on the relation between the biofilm formation capability of bacteria and the surface characteristics of materials. ${ }^{33}$ However, de Avila et al. ${ }^{34}$ showed that the hydrophilicity of ultraviolet (UV)-photofunctionalized titanium surfaces increases and the bacterial adherence and biofilm formation on such surfaces decrease. Decreased bacterial adherence to UV-photofunctionalized surfaces was attributed to surface modification of titanium during exposure to UV light, which leads to increased hydrophilicity. This increase in the hydrophilicity of plasma-treated surfaces is a well-known phenomenon and has been reported by various groups. ${ }^{35-37}$ Similarly,

Volume 6, Issue 1, 2016 

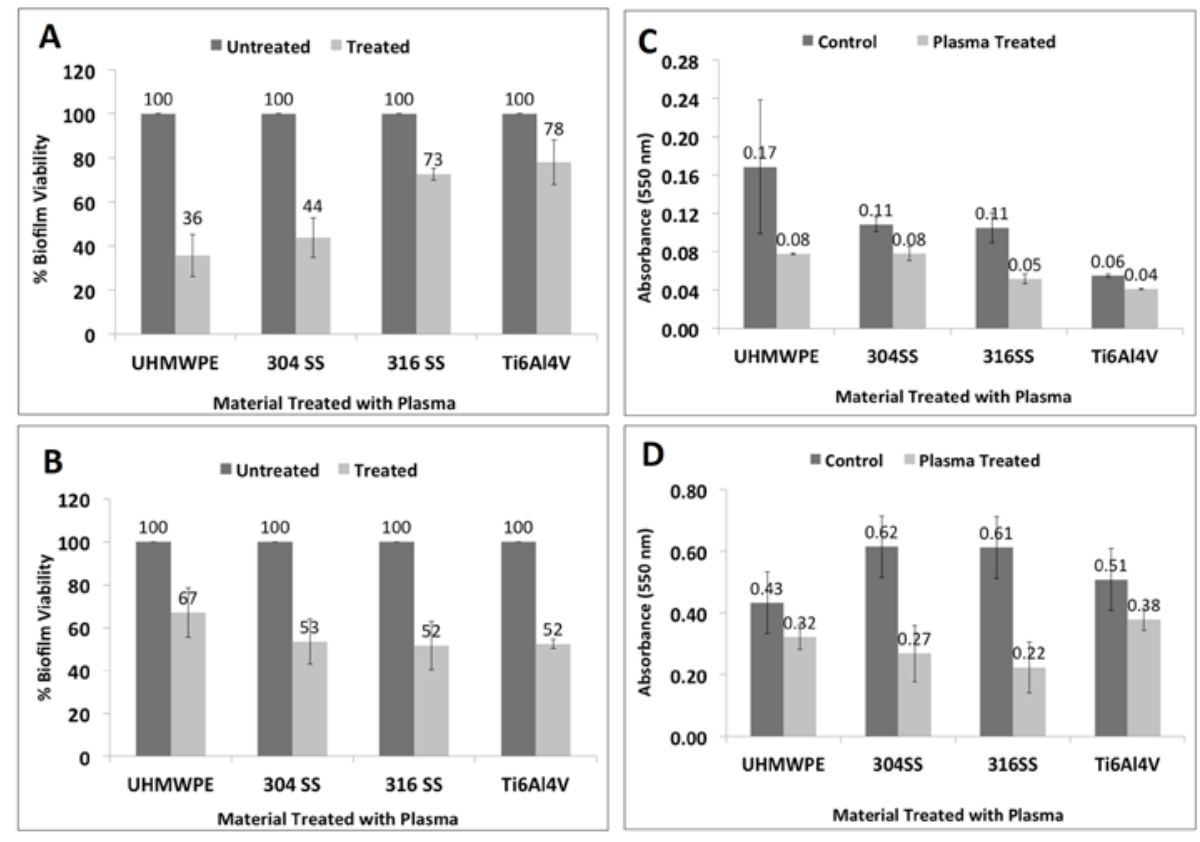

FIG. 6: A 10-minute plasma treatment of 304 stainless steel (SS), 316 L SS, $\mathrm{Ti}_{6} \mathrm{Al}_{4} \mathrm{~V}$, and ultrahigh-molecular-weight polyethylene (UHMWPE) discs before biofilm growth reduces Escherichia coli (A) and Staphylococcus aureus (B) biofilm formation on disc surfaces. Decreased extracellular polymeric substances of biofilms on discs, which were treated for 10 minutes with nonthermal plasma before biofilm growth, also indicates reduced biofilm formation of $E$. coli (C) and $S$. aureus (D).

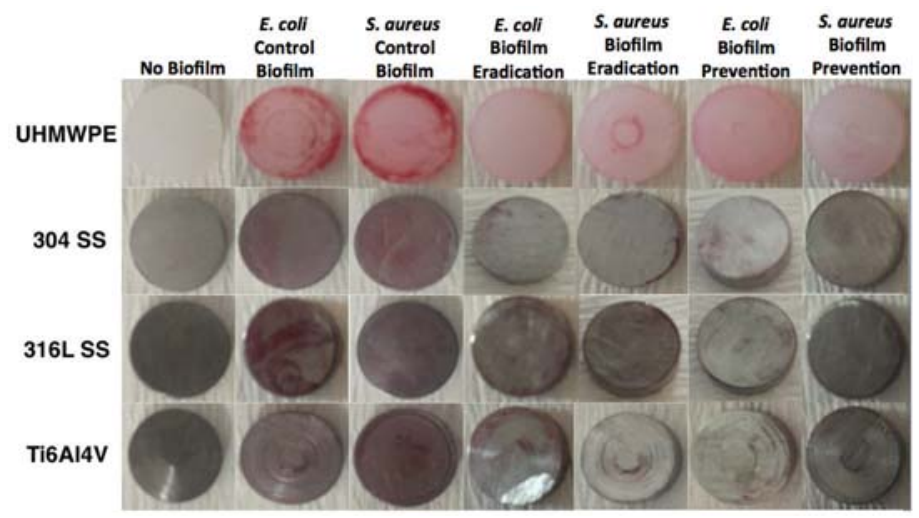

FIG. 7: The prevention of biofilm formation and the biofilm eradication effects of nonthermal, atmospheric dielectric barrier discharge air plasma treatment on 304 stainless steel (SS), 316L $\mathrm{SS}, \mathrm{Ti}_{6} \mathrm{Al}_{4} \mathrm{~V}$, and ultra-high-molecular-weight polyethylene (UHMWPE) material surfaces were macroscopically visualized using safranin dye, which stains extracellular polymeric substances. Compared with discs growing a biofilm, a lower intensity of safranin dye, which was used in biofilm eradication and prevention experiments, can be observed on the discs. 
we saw an increase in the hydrophilicity of 304 SS, 316L SS, Ti6Al4V, and UHMWPE surfaces, and this surface modification was attributed to the prevention of biofilm formation on the tested material surfaces, similar to UV-photofunctionalized titanium surfaces.

\section{CONCLUSION}

In this study we evaluated the biofilm eradication and the biofilm prevention capabilities of nonthermal, atmospheric air DBD plasma on $304 \mathrm{SS}, 316 \mathrm{~L} \mathrm{SS}$, Ti6A14V, and UHMWPE surfaces. For biofilm eradication experiments, 1-day-old biofilms of $E$. coli and $S$. aureus were grown on implant materials and then treated with DBD plasma. As for the prevention of biofilm formation, implant materials were first treated with DBD plasma, then biofilms were grown on the surfaces. Our results show that DBD plasma treatment of implant surfaces inactivates up to $>95 \%$ of biofilms on the implant surfaces. Moreover, biofilm formation on DBD plasma-treated implant material surfaces was retarded up to $50 \%$. The effects of plasma treatment on the eradication of biofilm and on the prevention of biofilm formation were also assessed in terms of the amount of EPS on the implant material surfaces. Our results reveal that plasma treatment disrupts the integrity of the biofilm during inactivation and can prevent biofilm formation. Eradication of bacterial biofilms on implant materials was attributed to reactive oxygen and nitrogen species generated during plasma treatment. On the other hand, we also showed increased hydrophilicity of implant material surfaces after DBD plasma treatment, which could be considered as a reason for the mechanism of prevention of the biofilm formation.

In conclusion, nonthermal, atmospheric air DBD plasma can be used to decontaminate common implant materials. Moreover, biofilm formation could be prevented to some extent on implant material surfaces previously treated with plasma. Therefore, nonthermal atmospheric plasma can be considered as a novel tool for the prevention and control of implant site infections.

\section{ACKNOWLEDGMENT}

This study was financially supported by the Department of Scientific Research Projects, İzmir Katip Çelebi University (BAP, 2014-1-FMBP-01).

\section{REFERENCES}

1. Lane WA. Some remarks on the treatment of fractures. Br Med J. 1895;1:861-3.

2. Hermawan H, Ramdan D, Djuansjah JRP. Metals for biomedical applications. In: Fazel R, editor. Biomedical engineering - from theory to applications. Rijeka, Croatia: InTech Publication; 2011. Available from: http://www.intechopen.com/books/biomedical-engineering-from-theory-to-applications/metalsfor-biomedical-applications.

3. Serro AP, Saramago, B. Influence of sterilization on the mineralization of Ti implants induced by incubation in various biological model fluids. Biomaterials. 2013;24(26):4749-60.

4. Affatato S, Bordini B, Fagnano C, Taddei P, Tinti A, Toni A. Effects of sterilization method on the wear of UHMWPE acetabular cups tested in a hip joint simulator. Biomaterials. 2002;23(6):1439-46.

Volume 6, Issue 1, 2016 
İbiş, Oflaz, \& Ercan

5. Jardini AL, Larosa MA, Bernardes LF, Zavaglia CAC, Maciel Filho R. Application of direct metal laser sintering in titanium alloy for cranioplasty. Proceedings of the 6th Brazilian Conference on Manufacturing Engineering; 2011 Apr 11-15; Caxias do Sul, Brazil.

6. Damani N. Manual of infection prevention and control. 3rd ed. Oxford: Oxford University Press; 2012.

7. Chen M, Yu Q, Sun H. Novel strategies for the prevention and treatment of biofilm related infections. Int J Mol Sci. 2013;14(9):18488-501.

8. Junter G-A, Thébault P, Lebrun L. Polysaccharide based antibiofilm surfaces. Acta Biomater. 2016;30:13-25.

9. Ta CA, Aranson JT. Mini review of phytochemicals and plant taxa with activity as microbial biofilm and quorum sensing. Molecules. 2015;21(1). pii: E29.

10. Marques CN, Davies DG, Sauer K. Control of biofilms with the fatty acid signaling molecule cis2-decenoic acid. Pharmaceuticals (Basel). 2015;8(4):816-35.

11. Veerachamy S, Yarlagadda T, Manivasagam G, Yarlagadda PK. Bacterial adherence and biofilm formation on medical implants: a review. Proc Inst Mech Eng H. 2014;228(10):1083-99.

12. Hota B. Contamination, disinfection, and cross-colonization: are hospital surfaces reservoirs for nosocomial infection? Clin Infect Dis. 2004;39(8):1182-9.

13. Lee A, Wang HL. Biofilm related to dental implants. Implant Dent. 2010;19(5):387-91.

14. Joshi SG, Cooper M, Yost A, Paff M, Ercan UK, Fridman G, Friedman G, Fridman A, Brooks AD. Nonthermal dielectric-barrier discharge plasma-induced inactivation involves oxidative DNA and membrane lipid peroxidation in Escherichia coli. Antimicrob Agents Chemother. 2011;55(3): 1053-62.

15. Zimmermann J, Dumler K, Shimizu, T, Morfill, GE, Wolf A, Boxhammer V, Schlegel J, Gansbacher B, Anton M. Effects of cold atmospheric plasmas on adenovirus in solution. J Phys D Appl Phys. 2011;44(50):505201.

16. Maisch T, Shimizu T, Isbary G, Heinlin J, Karrer S, Klampfl TG, Li YF, Morfill G, Zimmerman JL. Contact-free inactivation of Candida albicans biofilms by cold atmospheric air plasma. Appl Environ Microbiol. 2011:78(12):4242-7.

17. Elmoualij B, Thellin O, Gofflot S, Heinen E, Levif P, Seguin J, Moisan M, Leduc A, Barbeau J, Zorzi W. Decontamination of prions by the flowing afterglow of a reduced-pressure N2-O2 cold-plasma. Plasma Process Polym. 2012;9(6):612-8.

18. Ercan UK, Wang H, Ji H, Fridman G, Brooks AD, Joshi SG. Nonequilibrium plasma-activated antimicrobial solutions are broad-spectrum and retain their efficacies for extended period of time. Plasma Process Polym. 2013;10(6):544-55.

19. Poor AE, Ercan UK, Yost A, Brooks AD, Joshi SG. Control of multi-drug-resistant pathogens with non-thermal-plasma-treated alginate wound dressing. Surg Infect (Larchmt). 2014;15(3):233-43.

20. Yorsaeng S, Pornsunthorntawee O, Rujiravanit R. Preparation and characterization of chitosan-coated DBD plasma treated natural rubber latex medical surgical gloves with antibacterial activities. Plasma Chem Plasma Process. 2012;32(6):1275-92.

21. Monetta T, Scala A, Malmo C, Bellucci F. Antibacterial activity of cold plasma-treated titanium alloy. Plasma Med. 2011;1(3-4):205-14.

22. Bellebna Y, Tilmatine A. Application of dielectric surface barrier discharge for air disinfection. Acta Electrotech Inform. 2013;13(3):22-6.

23. Thomas FO, Corke TC, Iqbal M, Kozlov A, Schatzman D. Optimization of dielectric barrier discharge plasma actuators for active aerodynamic flow control. AIAA J. 2009;47(9):2169-78.

24. Kogelschatz U. Dielectric barrier discharges: their history, discharge physics, and industrial applications. Plasma Chem Plasma Process. 2003;23(1):1-46.

25. Corke TC, Post ML, Orlov DM. Single dielectric barrier discharge plasma enhanced aerodynamics: physics, modeling and applications. Exp Fluids. 2009;46(1):1-26.

26. Fridman G, Friedman G, Gutsol A, Shekhter AB, Vasilets VN, Fridman A. Applied plasma medicine. Plasma Process Polym. 2008;5(6):503-33. 
27. Oehmigen K, Hahnel M, Brandenburg R, Wilke C, Weltman KD, von Woedtke T. The role of acidification of atmospheric pressure plasma in liquids. Plasma Process Polym. 2010;7(3-4):250-7.

28. Kojtari A, Ercan UK, Josh S, Friedman G, Sensenig R, Tyagi S, Joshi SG, Ji H, Brooks AD. Chemistry for antimicrobial properties of water treated with non-equilibrium plasma. J Nanomedicine Biotherapeutic Discov. 2013;4(1):1000120.

29. Alkawareek MY, Algwari QT, Gorman SP, Graham WG, O’Connel D, Gilmor BF. Application of atmospheric pressure nonthermal plasma for the in vitro eradication of bacterial biofilms. FEMS Immunol Med Microbiol. 2012;65(2):381-4.

30. Fricke K, Koban I, Tresp H, Jablonowski L, Schröder K, Kramer A, Weltmann KD, von Woedtke T, Kocher T. Atmospheric pressure plasma: a high-performance tool for the efficient removal of biofilms. PLoS One. 2012;7(8):e42539.

31. Joaquin JC, Kwan C, Abramzon N, Vandervoort K, Brelles-Marino G. Is gas- discharge plasma a new solution to the old problem of biofilm inactivation? Microbiology. 2009;155(Pt 3):724-32.

32. Koban I, Holtfreter B, Hübner NO, Matthes R, Sietmann R, Kindel E, Weltmann KD, Welk A, Kramer A, Kocher T. Antimicrobial efficacy of non-thermal plasma in comparison to chlorhexidine against dental biofilms on titanium discs in vitro - proof of principle experiment. J Clin Periodontol. 2011;38(10):956-65.

33. Krasowska A, Sigler K. How microorganisms use hydrophobicity and what does this mean for human needs? Front Cell Infect Microbiol. 2014;4:112.

34. de Avila ED, Lima BP, Sekiya T, Torii Y, Ogawa T, Shi W, Lux R. Effect of UV-photofunctionalization on oral bacterial attachment and biofilm formation to titanium implant material. Biomaterials. 2015;67:84-92.

35. Shon WJ, Chung SH, Kim HK, Han GJ, Cho BH, Park YS. Peri-implant bone formation of non-thermal atmospheric pressure plasma-treated zirconia implants with different surface roughness in rabbit tibiae. Clin Oral Implants Res. 2014;25(5):573-9.

36. Dolci LS, Quiroga SD, Gherardi M, Laurita R, Liguori A, Ghedini E, Sanibondi P, Fiorani A, Calzà L, Colombo V, Focarete ML. Atmospheric non-thermal plasma surface modification of poly(L-lactic acid) electrospun nanofibers affects scaffold hydrophilicity and fibroblast morphological response. Proceedings of 21st International Symposium on Plasma Chemistry; 2013 Aug 4-9; Cairns, Australia.

37. Park JH, Olivares-Navarrete R, Baier RE, Meyer AE, Tannenbaum R, Boyan BD, Schwartz Z. Effect of cleaning and sterilization on titanium implant surface properties and cellular response. Acta Biomater. 2012;8(5):1966-75.

Volume 6, Issue 1, 2016 